\title{
Research on Safety Management of Construction Engineering Personnel under "Big Data + Artificial Intelligence"
}

\author{
Xizhe Yi, Jiaye Wu \\ Name of Organization, Sichuan University of Science \& Engineering, Zigong, China \\ Email: 1024228398@qq.com
}

How to cite this paper: Yi, X. Z., \& Wu, J. Y. (2020). Research on Safety Management of Construction Engineering Personnel under "Big Data + Artificial Intelligence". Open Journal of Business and Management, 8, 1059-1075.

https://doi.org/10.4236/ojbm.2020.83067

Received: March 5, 2020

Accepted: April 10, 2020

Published: April 13, 2020

Copyright $\odot 2020$ by author(s) and Scientific Research Publishing Inc. This work is licensed under the Creative Commons Attribution International License (CC BY 4.0).

http://creativecommons.org/licenses/by/4.0/

\begin{abstract}
Under the background of the information age, traditional engineering safety management is undergoing a profound change. The introduction of big data and artificial intelligence technologies in construction project safety management is becoming increasingly widespread. Based on literature analysis, combined with artificial intelligence and big data in construction safety management in the application is still in the primary stage, especially in applied research personnel safety management rarely literature. A review of the exploration and application of construction engineering safety management under the combination of artificial intelligence and big data, the analysis and summary of the current status and trends of the two technologies in construction engineering safety management, and the design of Based on artificial intelligence engineering personnel safety management of large data systems architecture, enrich and improve construction safety of personnel management concepts and technical means.
\end{abstract}

\section{Keywords}

Management Science, Engineering

\section{Introduction}

For a long time, the number of construction engineering safety accidents and workers' casualties has been high. Based on traditional thinking, the safety management of construction engineering personnel is dominated by managers' own experience, skills, knowledge and ideas, and adopts low-efficiency manual supervision. This shows that the traditional personnel safety management has strong subjectivity, high input of safety resources but poor management effect. 
As the most active factor in the construction process, people have the characteristics of strong initiative, frequent action, fast flow speed and wide activity space, so it is obvious that the limited human input cannot play a good role in ensuring the safety of personnel. Therefore, the traditional management method has the disadvantages of high human cost and low supervision efficiency, which cannot achieve the purpose of effective management. The traditional personnel safety management urgently needs to innovate and change the current mode.

The emerging technology wave represented by big data and artificial intelligence provides technical support for the transformation and upgrading of safety management of construction engineering personnel. A great change of safety management concept, management mode and management method is underway, and safety management is moving towards intelligent analysis, scientific decision-making and refined management. First of all, construction project safety management combines big data technology, with the prominent advantage of assisting safety management personnel in scientific decision-making and accurate prediction (Yu et al., 2019; Yang et al., 2017; Ma et al., 2015b); in addition, personnel safety management combines artificial intelligence to promote the intelligent development of personnel safety management process (Han et al., 2018; Zeng, 2017; Wang \& Wu, 2012b). They complement each other, assist participants in all stages to achieve the perfection from the bottom application to the top design, and jointly innovate traditional personnel safety management methods.

At present, the domestic research on the combination of engineering construction safety management and two technologies has been carried out (Leng \& Hu, 2018; Zhang et al., 2018; Zeng, 2014), but most of them are the combination of single technology and safety management, and the fusibility of the two has not attracted enough attention of the academic community; in addition, the relevant summary and system design of personnel safety management are still not seen in the literature. In this context, this paper summarizes the safety management of construction personnel in the era of artificial intelligence and big data, and designs the big data system architecture of safety management of engineering personnel based on artificial intelligence, aiming to provide direction for subsequent research.

\section{Interpretation of Basic Concepts}

\subsection{Basic Concepts of Big Data}

The concept of big data was first proposed by McKinsey in 2011 (Liu \& Zhang, 2014), which refers to a data set that cannot be effectively acquired, managed and processed by using traditional databases or software within a limited time. There is a general consensus on its characteristics, namely, the 4 "V" characteristics of large capacity, many types, high speed and low value density (Liu \& Zhang, 2014). With the development of Internet of things technology, massive data in all aspects of engineering construction has been recorded through vari- 
ous equipment carriers, which has the characteristics of large amount of data, various types, fast production speed and low value density.

Big data inheritance data mining and analysis, close combination of cloud computing technology to solve the traditional data processing methods cannot complete the acquisition, storage, management, analysis of massive data, which provides strong technical support and scientific decision-making basis for all aspects of personnel security management. With the development of big data technology, the ability of optimization, prediction and intelligent decision-making has become the label of big data technology (Liu \& Zhang, 2014). Therefore, the combination of big data technology and personnel safety management is the inevitable choice to get rid of traditional subjective thinking and promote scientific decision-making.

\subsection{Basic Concepts of Artificial Intelligence}

Artificial intelligence is a subject that studies the laws of human intelligence and uses machines to simulate human intelligence, so as to simulate, extend and expand human intelligence (Leng \& $\mathrm{Hu}, 2018$ ). It originated from image recognition (Rao et al., 2019) and has been developed since it was put forward in 1956. Professor Nelson of the artificial intelligence research center of Stanford University defines artificial intelligence as: "artificial intelligence is a subject about knowledge, how to express knowledge and how to acquire and use knowledge" and according to Professor Winston of Massachusetts Institute of technology, "Artificial intelligence is to study how to make computers do intelligent work that only human can do in the past". This reflects that artificial intelligence aims at computer being competent for human's work, freeing people from simple labor, thus saving more human costs. Therefore, the combination with the construction industry will further accelerate the intelligent development of engineering management.

In the process of personnel safety management, due to human subjectivity, static and local management methods cannot achieve the desired results. According to literature research, image recognition technology has been widely used in construction safety management (Zeng, 2017; Han et al., 2016; Hu \& Su, 2018). The development of image recognition technology and the emergence of intelligent monitoring based on image recognition technology (Huang et al., 2015) make the real-time, dynamic, efficient and accurate method of personnel intelligent supervision possible.

\section{Analysis of Personnel Safety Management under "Big Data + Artificial Intelligence"}

The application of the combination of artificial intelligence and big data in the construction safety management is still in the primary stage, and there are few literatures about the combination of them, especially in the application of personnel safety management. In view of this, by summarizing the exploration and 
application of construction safety management in the field of artificial intelligence and big data, this paper analyzes the combination trend of the two technologies in personnel safety management, and makes up for the lack of traditional construction personnel management concepts and means.

\subsection{Summary of Personnel Safety Management under Big Data}

Engineering safety management ensures the safe operation of the project, shoulders social and economic responsibilities, and cannot be ignored. According to the literature review, the application of big data technology in security management is the most extensive, involving field management, structural security, disaster prevention, security management in operation period, etc.

Big data is different from traditional manual experience analysis. The core feature of big data technology is to find and use the hidden rules by analyzing massive data, so as to improve the safety management of construction site with data science. S. Y. Guo (Guo et al., 2016) established a database of workers' behaviors to collect, classify and store unsafe behaviors of workers, which laid a data foundation for later research; Beijing Zhangjiakou high speed railway Qinghuayuan tunnel (Zhi et al., 2018) used Bim and big data to promote parameter curve analysis and decision-making, based on big data of construction site safety, used three-dimensional visual analysis of BIM to realize safety early warning visualization and on-site real-time monitoring; big The management of large machinery is always the key link of safety management in construction site. Although large machinery is the guarantee of project progress, it is also one of the sources of frequent accidents. Jin Wei et al. (Jin et al., 2018) comprehensively used the Internet of things and big data to build a tower crane safety management system for intelligent construction sites; Heng Li (Li et al., 2016) proposed a site safety status prediction method through historical data mining and analysis, combined with RTLS and dtmc models. The above research shows that big data has the ability of real-time dynamic, scientific and efficient analysis, prediction and auxiliary decision-making in the process of construction safety management, but focuses on the field mechanical layout and visual monitoring research, showing the lack of research on personnel safety management.

In addition, the combination of big data and AI will produce strong flexibility, adaptability and learning ability, which is of great significance for innovation of traditional safety management methods. Wang Wen et al. (Wang et al., 2018) developed the tunnel safety monitoring system by deeply learning the video big data of the tunnel installation site through convolution neural network, which realized the intelligent management of risk early warning and tunnel abnormal event monitoring; Kinam Kim (Kim et al., 2017) developed the machine vision hazard avoidance system through the machine learning of the construction big data of the site to remind workers of potential safety risks. The above literature provides a case study and reference for the feasibility of the application of big data combined with $\mathrm{AI}$ in engineering safety management, and affirms the prac- 
ticability and efficiency of video monitoring in personnel safety management and risk early warning intelligent management. This paper provides guidance for the study of safety management of construction personnel.

The health monitoring platform based on big data has also been applied in practice. Tu Chengfeng et al. (Tu et al. 2017) studied multi-scale correlation analysis, abnormal data mining and analysis, and time-varying reliability based on long-term health monitoring big data analysis, developed an abnormal data mining engine, and built a bridge health monitoring big data analysis and monitoring service platform based on cloud computing; Wu zhixia et al. (Wu et al., 2018) developed a bridge health based on big data collection and processing The monitoring and maintenance platform has been successfully applied to the structural safety management of several bridges in Shanghai. The health monitoring platform based on big data is formed on the basis of the accumulation and development of health monitoring big data, which is a more in-depth application of big data technology combined with safety management. Big data health monitoring platform is an important platform for data accumulation, storage, extraction, sharing, analysis and evaluation, and provides rich and reliable data resources support for subsequent application research.

In addition, the core of safety monitoring big data is to predict ( $\mathrm{Ma}$ et al., 2015a) and calculate the incident rate, which meets the requirements of building fire safety. Based on the historical data of buildings and fires, Suzhou fire brigade adopts BP neural network and other algorithms for data machine learning to establish the "eye of fire" fire risk prediction system, which improves the efficiency of building fire prevention by 6 - 8 times (Zhu, 2017); Li Yongfeng and others (Li et al., 2018b) build Hadoop cluster, and design the building fire prediction system by combining wireless sensor network, AI and big data processing technologies; Xu Adamancy et al. (Xu et al., 2019) built a fire dynamic risk assessment model based on big data platform. All the above researches are aimed at realizing a dynamic, efficient and accurate intelligent disaster assessment method. In addition to fire safety, prediction characteristics are also used for structural safety. Ren Xiaoyu et al. (Ren et al., 2018) integrated random forest and gbdt algorithm, built a big data prediction model of building cracks, assisted the decision-making of building cracks processing, and ensured the structural safety of buildings. In terms of safety risk assessment, Guo Wenjuan (Guo, 2019) based on the big data analysis of construction accidents in China from 2013 to 2017 for construction safety management Based on big data and other Internet technologies, Liao Zhipeng et al. (Liao et al., 2018) established a web accident database and risk assessment information system for tunnel operation; C. Zhou (Zhou et al., 2018) combined with high-dimensional data mining technology and complex network technology for safety and performance assessment during shield construction of Wuhan Yangtze River tunnel. The above research provides a reference for the application of big data prediction ability in construction safety management from the theoretical and application level, and provides a reference 
for the design of early warning mechanism in the process of construction personnel safety management.

This section combs and analyzes the relevant documents of engineering safety management under big data (see Figure 1), and summarizes the application of big data and AI in engineering safety management at home and abroad, especially the research based on personnel safety management has been carried out, but the number is small, reflecting that the safety management of construction personnel is intelligent, the process of informatization is slow, and the attention from all walks of life is insufficient. Secondly, the application of big data mainly focuses on site management, structural safety, disaster prevention, and safety management during operation period, which has been effectively applied. However, the construction management, especially the safety management of construction personnel, is very limited, resulting in the low scientific and technological content of safety management of construction personnel. Big data technology

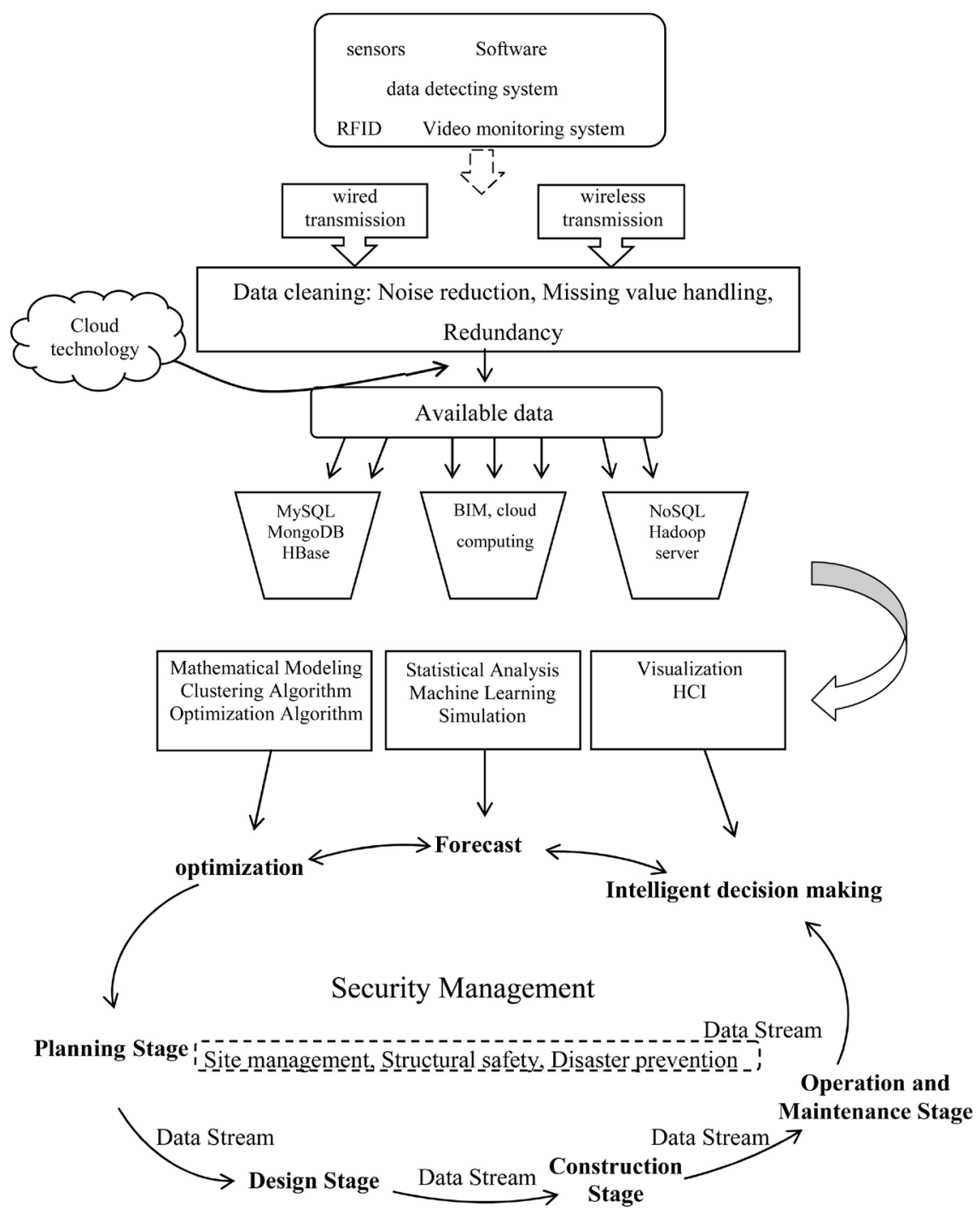

Figure 1. Engineering safety management under the combination of big data. 
combined with AI to improve the intelligent level of safety management of construction personnel should be an important and continuous research direction in the future.

\subsection{Summary of Personnel Safety Management under Artificial Intelligence}

AI technology is more flexible, and the combination of BIM (Leng \& Hu, 2018), big data (Rao et al., 2019), Internet of things technology (Han et al., 2018) has achieved certain results in both theory and practice. Through literature analysis, it is considered that the application prospect of artificial intelligence technology represented by image recognition in construction safety management is wide, and the real-time, accuracy and intelligent characteristics of related technologies are very in line with the requirements of construction personnel safety management (Han et al., 2018).

Zhang Yunyi et al. (Zhang et al., 2018) believe that cloud, big data, Internet of things and BIM Technology are indispensable. Only when they are fully integrated can they jointly play a role in serving construction project construction and management. Thus, a unified integrated application framework of various technologies is established. This has laid a theoretical framework foundation for the integration of multiple emerging technologies to serve engineering management, but the integration framework does not include artificial intelligence technology and fails to make full use of the characteristics of artificial intelligence technology, such as flexibility and strong adhesiveness, so it needs to be further improved; Wang Yaowu et al. (Wang \& Wu, 2012a, 2012b) have built a theoretical framework for intelligent construction and described the key technologies, which is more advanced. The construction management of technology integration provides theoretical and application guidance, but the research is relatively macro, and the details of personnel management are not discussed in depth; Han Yu et al. (Han et al., 2018) emphasize interdisciplinary integration and innovation, combing the solutions of intelligent construction site according to the two types of workers oriented and engineering oriented, and image recognition technology in workers' intelligent management, equipment management, vehicle management, and prevention The safety inspection of protective facilities has been effectively applied. This has a good foresight for the safety management of construction personnel, and has a good inspiration and guidance for the deep integration of big data and artificial intelligence. In addition, document (Li et al., 2018a; Li et al., 2019; Pei \& Zhang, 2019; Liu et al., 2019; Ma et al., 2018; Huang $\& \mathrm{Li}, 2017$ ) focuses on image recognition in defect detection (Li et al., 2018a), vehicle management (Li et al., 2019; Pei \& Zhang, 2019), safety inspection of protective facilities (Liu et al., 2019; Ma et al., 2018), disaster monitoring during operation (Huang \& Li, 2017), etc. The contribution of these studies is to provide necessary innovative guarantee means for the structural safety in the construction process and later maintenance stage, aiming to avoid the occurrence of 
safety accidents by ensuring the structural safety of the project. However, the exclusive comprehensive safety management system for construction personnel is very limited, which needs to be supplemented and improved, which further proves that image recognition in the construction safety management the feasibility, effectiveness and the necessity of innovating the safety management system of construction personnel.

Foreign research on the combination of artificial intelligence and construction safety management also focuses on the application of image recognition. Gamze et al. (Dogan et al., 2017) used the image processing technology based on artificial neural network to realize the detection method of the mechanical properties of non-destructive concrete. Manh et al. (Phung et al., 2017) improved the visual algorithm based on the existing infrastructure monitoring, improved the detection speed and made it easy for subsequent research. Chen et al. (Zhu et al., 2017) proposed a new framework of using camera to apply visual tracking technology to the detection of construction teams and equipment, for the inspection of workers' equipment and on-site mechanical equipment. This is a rare article that can link intelligent monitoring with human and machine management. It has reference significance for the design of this system, but it stops at the research of detection accuracy, and does not further deepen the research on personnel safety management. Martin et al. (Yu et al., 2017) based on the real-time image skeleton parameterization method to identify the precursors of unsafe behaviors of construction workers and make prevention, but the convenience of the equipment used still needs to be improved. It is not hard to see that foreign research in this field is mostly focused on building defect disaster management, while the number of research on personnel safety management is limited, and stays on the basic functions of object detection and simple body recognition, and does not dig deep into the further application of image recognition in personnel safety management, and the functions are scattered, the integration is low, and the implementation methods are different. As a result, the difficulty of managers' learning increases and the threshold of equipment use increases, which leads to the increase of application cost of management system and hinders the subsequent application and promotion. The above literature further clarifies the function orientation and system design of the system, providing theoretical basis and practical experience guidance.

This section combs and analyzes the literature related to the summary of personnel safety management under artificial intelligence (see Figure 2). It summarizes that the research on the combination of artificial intelligence technology in the process of construction management at home and abroad has gradually deepened, mainly focusing on the quality defect detection, disaster detection and construction site management of image recognition combined with other emerging technologies, so the research on personnel management is not comprehensive and in-depth. Secondly, human based management has not been fully integrated into the process of intelligent management, and the emphasis on 


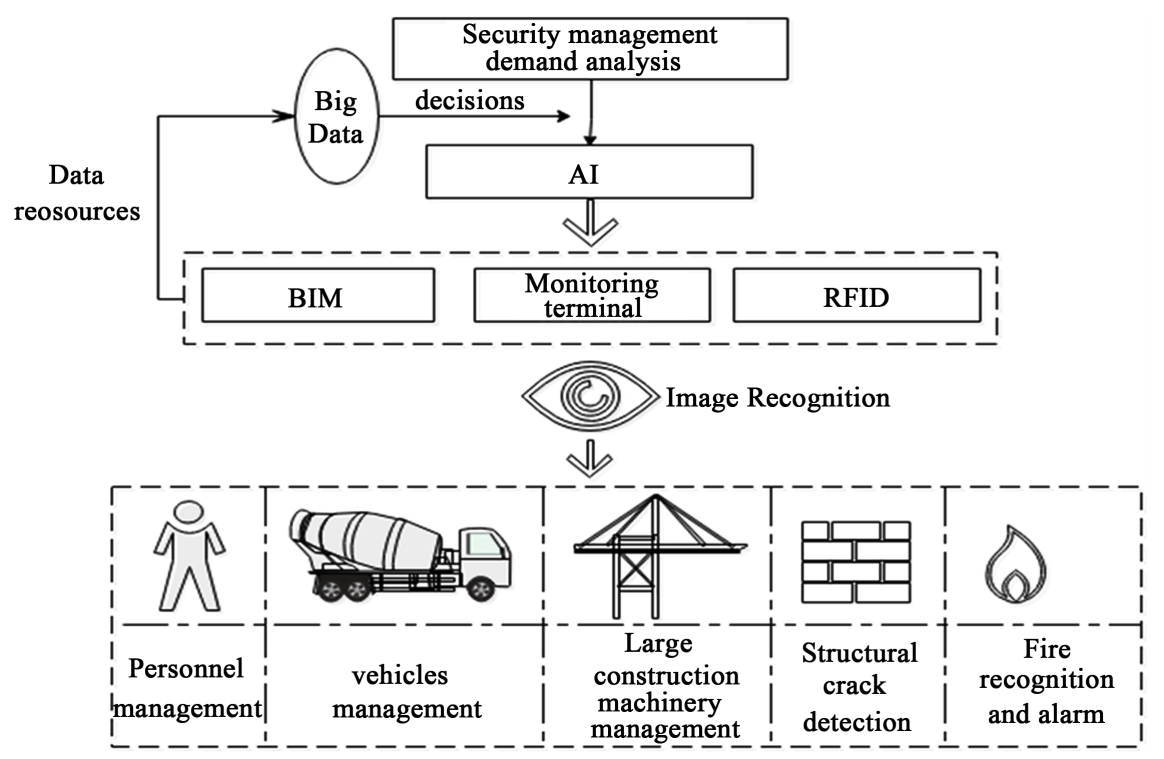

Figure 2. Engineering safety management under the combination of artificial intelligence.

people as one of the determinants of safe construction is far from enough; finally, image recognition has shown a strong auxiliary ability in the process of construction safety management, which should be an important direction in the future, and the training resources required for image recognition will be collected by big data technology. The combination of the two will further promote the intelligent development of construction personnel safety management.

\subsection{Analysis of the Combination of Artificial Intelligence and Big Data}

Due to the strong initiative of people in the construction process of the project, the safety management of personnel has complexity and particularity, and the management difficulty increases. Therefore, at present, it is impossible to have a technology or a mode to run through management. The organic combination of multi-disciplinary, multi domain and multi cutting-edge technologies is the inevitable requirement for the scientific and efficient realization of management objectives. Based on the literature analysis, this paper discusses the application direction of big data combined with artificial intelligence in personnel safety management.

There is a hierarchical relationship between big data and artificial intelligence, but they influence and support each other (see Figure 3). Big data and cloud computing provide sufficient data resources and computing power for the implementation of artificial intelligence core algorithm. Through in-depth learning of data resources, artificial intelligence constantly optimizes learning experience, and finally gets the intelligent application that meets the requirements. Due to the high dependence of AI on the quantity and quality of data resources, the powerful data collection, processing and storage capabilities of big data technology will provide rich and reliable data accumulation and training resources for 


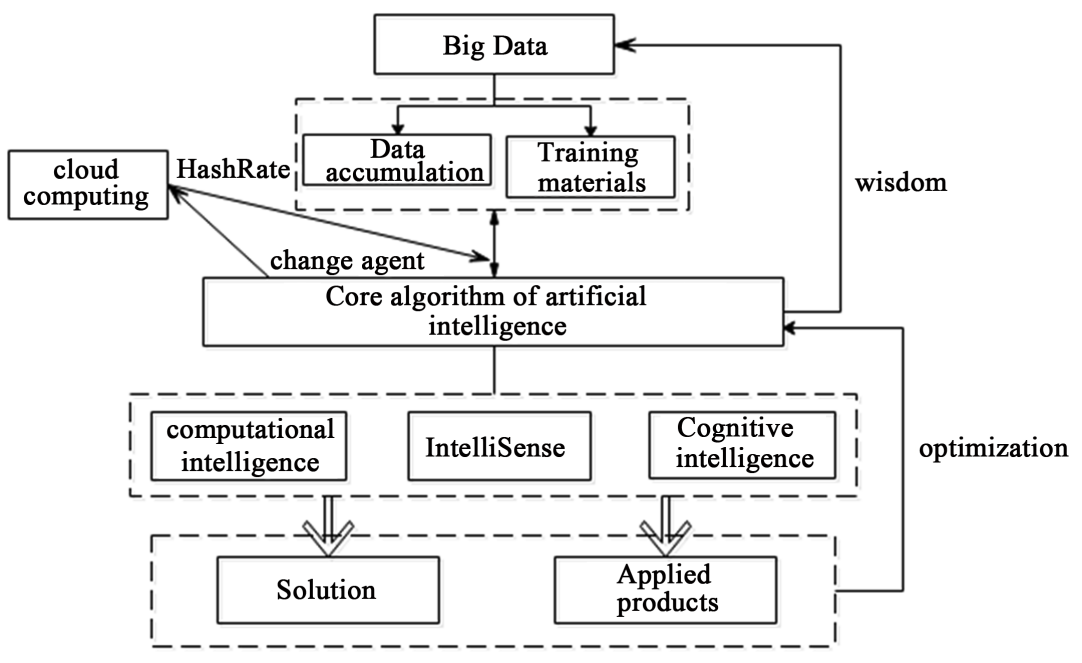

Figure 3. Analysis on the combination of big data and artificial intelligence.

AI, while the development of cloud computing and GPU meet the requirements of AI computing power; AI endows big data intelligence with core algorithms, and the core of big data makes With the development and application of artificial intelligence technology, value has shown great potential, making data serve users, and also providing change drivers for cloud computing. Therefore, artificial intelligence needs big data technology support, big data needs to be combined with artificial intelligence to form upper application and penetrate into various fields.

\subsection{Personnel Safety Management under "Big Data + Artificial Intelligence"}

Through the analysis of the combination of big data and artificial intelligence, and the literature analysis of the combination of construction safety management with big data and artificial intelligence technology, it is considered that the advantage of big data in personnel safety management is that it can effectively collect the data required for management and effectively process it to obtain reliable data for the safety management personnel to analyze at any time, and then to the artificial intelligence program design requirements. The important connection between big data and artificial intelligence is to use the ability of big data technology collection, processing and storage to obtain the required training materials. In addition, according to the literature research, the artificial intelligence technology represented by image recognition undertakes the training resources or decision-making needs provided by big data technology, develops the application programs required by supervision, and combines with intelligent monitoring to monitor the overall, real-time and accurate behavior of construction personnel, which can also improve supervision efficiency and safety while reducing personnel investment. Meet the requirements of real-time, accuracy and visualization for personnel safety management; at the same time, continue to play their respective advantages. Big data enables managers to reduce their 
dependence on subjective experience, scientifically predict the probability of occurrence of events and judge the safety status, so as to assist managers to make decisions based on data resources; artificial intelligence technology plays a role of demand realization tool, generates reliable program applications, and improves the quality of big data decision-making through its own development and optimization. Both of them have their own advantages and are related to each other, so as to promote the transformation and upgrading of traditional personnel safety management.

To sum up, the personnel safety management under "big data + artificial intelligence" proposed in this paper is based on the analysis of current relevant research, the enrichment and development of construction safety management theory, the innovation of traditional personnel safety management, and the interconnection with advanced concepts such as intelligent construction site and intelligent construction.

\section{Architecture Design of Big Data System for Safety Management of Construction Personnel Based on Artificial Intelligence}

Based on the above research and in-depth analysis of the safety management needs of construction personnel, the design concept of this paper is derived from the concept of intelligent construction site system architecture (Han et al., 2018) proposed by $\mathrm{Han} \mathrm{Yu}$, which conforms to the three connotation characteristics of more thorough perception, more comprehensive interconnection and more in-depth intelligence, and is supported by the design sense layer, transmission network layer and management application layer respectively. At the same time, based on the above research, the design concept of this paper is based on the concept of intelligent construction site system architecture (Han et al., 2018) proposed by $\mathrm{Han} \mathrm{Yu}$, in-depth analysis of the safety management needs of construction personnel, proposed the construction personnel safety management big data system architecture design based on artificial in telligence, as shown in Figure 4.

The device sensing layer includes various information collection and feedback devices, including various sensors, positioning modules, electronic tags and monitoring devices. The equipment perception layer is responsible for transmitting and feeding back personnel data information to the safety management big data platform for managers to analyze and make decisions, formulate scientific and reasonable safety management requirements, and adjust the site layout at any time. At the same time, as the equipment carrier in the management application layer, it enables the intelligent application assistant managers to complete the supervision of the construction personnel, so as to reduce the human investment and improve the supervision efficiency.

The transmission network layer includes all kinds of wired and wireless transmission modes such as Bluetooth, broadband, mobile communication, etc. 


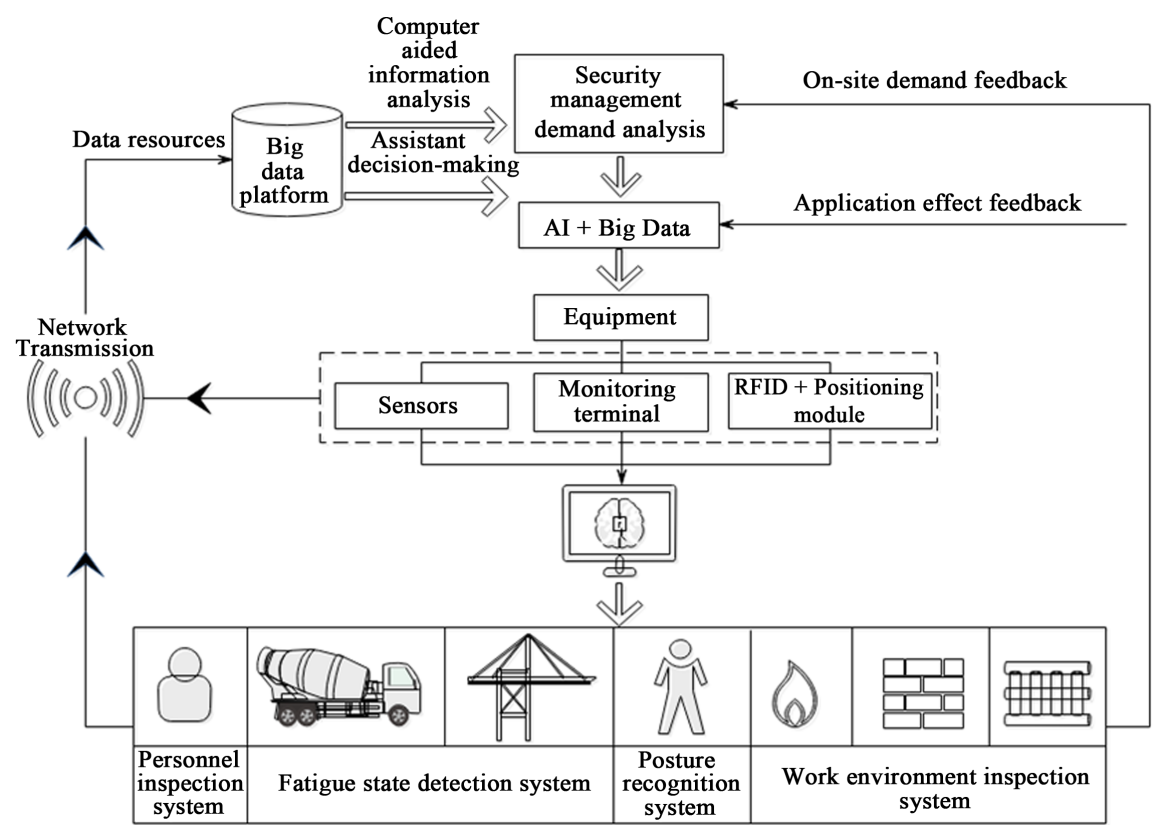

Figure 4. Big data system architecture of safety management for construction workers based on AI.

It is a bridge for field, application and management personnel to communicate with each other to realize information transmission and exchange.

The management application layer is the core technology layer of the system, including various intelligent application results under the combination of artificial intelligence technology and big data technology. On the basis of literature research, this paper analyzes the shortcomings of traditional personnel safety management methods, and summarizes the needs of personnel safety intelligent management; personnel safety intelligent management should achieve better safety management effect and higher supervision efficiency with less human investment and lower cost resource investment. Design "personnel inspection system", "fatigue state detection system", "body state recognition system" and "work environment inspection system".

It is considered that the construction personnel have the characteristics of strong initiative, frequent action, fast flow speed and wide activity space in the construction process, while the safety management personnel in the construction site have the limitations of quantity and energy, which cannot be comprehensive. Therefore, the contradiction between the characteristics of labor personnel in the construction process and the limitations of safety management personnel has existed for a long time. In order to achieve real-time visualization and intelligent monitoring effect, the above contradiction can be effectively solved by combining the image recognition technology which is widely used at present. Based on the training data provided by big data technology, in-depth neural network learning is carried out to provide specific intelligent monitoring application for different system requirements, real-time monitoring and identi- 
fication of personnel dynamics. At the same time, the decision-making ability of big data technology is used to analyze the safety management needs of personnel and make relevant decisions.

\subsection{Personnel Inspection System}

First of all, the system can identify the construction personnel through face recognition technology to complete daily attendance, access control management, professional equipment use authority inspection and other tasks. In order to prevent the occurrence of slack work, at the same time, to prevent unqualified personnel from operating equipment and reduce the safety accidents caused by illegal operation;

In addition, the use of object recognition and pedestrian recognition technology, to achieve construction personnel safety equipment inspection, such as safety helmet, safety belt and other safety equipment wear inspection, safety risk control in advance.

\subsection{Fatigue State Detection System}

Fatigue state detection system is mainly used for fatigue state detection of on-site vehicles and tower crane drivers. It is to collect the size and position of the driver's retinal pupil image with the camera, establish the mathematical model of the driver's fatigue driving, analyze and judge the locomotive driver's on duty look, when there are frequent blinking, closing eyes and other obvious fatigue driving situations, alarm the driver, and send the danger notice to the remote management personnel, so that the manager can handle it in time To avoid accidents.

\subsection{Posture Recognition System}

The body recognition system includes behavior ability inspection and unsafe behavior recognition of construction personnel. First of all, the behavior ability inspection of construction personnel is to identify the operation actions of employees before operation. If the angle change interval is consistent with the unqualified behavior information recorded in the behavior database, the angle of bone node is immediately determined to be inconsistent with the requirements. The physiological condition of the worker is abnormal, and the worker is not suitable for construction operation at present, and an alarm is given to realize prior control.

According to Heinrich's "safety pyramid" principle, unsafe behavior identification module must eliminate daily unsafe behavior and unsafe state in order to prevent death and serious injury accidents; whether it can eliminate daily unsafe behavior and unsafe state depends on whether the daily management is in place. Therefore, people's unsafe behavior is the decisive factor that affects safety production and leads to safety accidents. Meanwhile, big data technology is used to collect unsafe behavior training data, and image recognition technology is used to assist managers to stop unsafe behaviors of construction personnel. 


\subsection{Operating Environment Inspection System}

The work environment inspection system can assist the management personnel to inspect the construction environment by using video monitoring terminal based on image recognition and at the same time of manual inspection, and find hidden and difficult to check unsafe points, such as structural crack area, forbidden fire source area, protective fence, scaffolding and other areas with defective protective facilities, so as to improve the efficiency and accuracy of management supervision and ensure the safety of the staff construction.

All of the above systems are designed to assist managers to improve the efficiency of supervision and the accuracy of unsafe event identification. The related functions can also be achieved by human resources. However, in the face of dense and repeated shape of components, human beings have poor ability to find abnormal points, and the continuous supervision efficiency will be significantly reduced. Therefore, the limitation of human beings and the limitation of human investment are the driving force for the innovation of personnel safety management.

\section{Advantages and Disadvantages}

The advantage of big data system of safety management of construction personnel based on artificial intelligence is that it can complete a variety of cyclic structures, such as data collection feedback, application layer effect feedback, etc., so that the system itself can be continuously optimized. Intelligent application forms an intelligent system, so as to achieve better safety management effect with less human investment and lower cost resource investment, and further promote the traditional personnel safety management to intelligent.

The disadvantage is that no matter in literature research or in this study, computer terminals are mostly used in the system application layer, and the safety management of construction engineering personnel needs to go deep into the site. Computer terminals, especially desktop computer terminals, obviously hinder the application and promotion of the system due to the inconvenience of mobile; while mobile terminals such as hand-held computers are convenient for mobile, but the interface is less suitable for connecting sensors and subject to software. It is difficult to redevelop the hardware performance. Based on the existing research and implemented functions, this paper analyzes the needs of personnel safety management and designs the system framework, which is not comprehensive enough.

\section{Conclusion and Prospect}

1) The contribution of this paper is to summarize the engineering safety management under the big data technology and artificial intelligence technology, analyze the safety management requirements of construction personnel, establish the big data system architecture of construction personnel safety management based on artificial intelligence based on the intelligent site system frame- 
work, propose the concept of combining big data technology with image recognition, and solve the problem of real-time identification of construction personnel behavior problems, updating backward technical means, overcoming the disadvantages of traditional safety management.

2) In the process of innovation and development, personnel safety management also needs to attach great importance to the deep integration of equipment research and development, big data, artificial intelligence and other cutting-edge technologies, so as to meet the mobile convenience requirements of detection equipment on the project site. At the same time, personnel safety management involves a wide range of contents and needs, and the possible innovation in the future is to further investigate the micro equipment terminals that meet the engineering application needs, and strive to strengthen the innovation research of the construction personnel safety management system and the practical research of its technical realization mode, so as to improve the shortcomings of this research.

\section{Conflicts of Interest}

The authors declare no conflicts of interest regarding the publication of this paper.

\section{References}

Dogan, G., Arslan, M. H. et al. (2017). Concrete Compressive Strength Detection Using Image Processing Based New Test Method. Measurement, 109, 137-148. https://doi.org/10.1016/j.measurement.2017.05.051

Guo, S. Y., Ding, L. Y., Luo, H. B., \& Jiang, X. Y. (2016). A Big-Data-Based Platform of Workers' Behavior: Observations from the Field. Accident Analysis and Prevention, 93, 299-309. https://doi.org/10.1016/j.aap.2015.09.024

Guo, W. J. (2019). Risk Assessment Model for Building Construction under the Background of Big Data. Bulletin of Science and Technology, 35, 198-201.

Han, Y., Sun, H., Li, Y H., \& You, S. D. (2018). Architecture and Implementation of Smart Site System. Science \& Technology Progress and Policy, 35, 107-111. https://doi.org/10.1201/9780429429934-6

Han, Y., Zhang, J. J, Sun, H., Yao, J. Y., \& You, S. D. (2016). Design and Implementation of Intelligent Safety Inspection System for Construction Workers Based on Image Recognition. Journal of Safety Science and Technology, 12, 142-148.

Hu, Y. F., \& Su, Z. M. (2018). Application of Construction Safety Knowledge Ontology in Construction Site Safety Monitoring System. Modern Electronics Technique, 41, 91-95.

Huang, H. W., \& Li, Q. T. (2017). Image Recognition for Water Leakage in Shield Tunnel Based on Deep Learning. Chinese Journal of Rock Mechanics and Engineering, 36, 2861-2871.

Huang, K. Q., Chen, X. T., Kang, Y. F., \& Tan, T. N. (2015). Intelligent Visual Surveillance: A Review. Chinese Journal of Computers, 38, 1093-1118.

Jin, W., Zeng, B., Dong, L., Qu, H. B., \& Wang, J. Q. (2018). A Study on Big Data of Tower Crane Safety Management for Smart Construction. Construction Mechanization, 39, 22-27. 
Kim, K., Kim, H., \& Kim, H. (2017). Image-Based Construction Hazard Avoidance System Using Augmented Reality in Wearable Device. Automation in Construction, 83, 390-403. https://doi.org/10.1016/j.autcon.2017.06.014

Leng, S., \& Hu, Z. Z. (2018). A Review of BIM-Based Artificial Intelligence Methods. Journal of Graphics, 39, 797-805.

Li, H., Yang, X. C., Wang, F. L., Rose, T., Chan, G., \& Dong, S. (2016). Stochastic State Sequence Model to Predict Construction Site Safety States through Real-Time Location Systems. Safety Science, 84, 78-87. https://doi.org/10.1016/j.ssci.2015.11.025

Li, J. C., Zhang, C. B., Chai, X. S., \& Xue, F. (2018a). Research on Crack Detection System of Tunnel Lining Based on Image Recognition Technology. Railway Engineering, 58, 20-24.

Li, Y. F., Gao, Y., Huang, J., \& Yun, Z. H. (2018b). Research on the Fire Detection System in Tibetan Ancient Buildings Based on Hadoop. Fire Science and Technology, 37, 219-222.

Li, Y. H., Han, Y., Yang, Y. X., Rui, Y., \& Sun, R. (2019). Design and Implementation of Supervision System for Muck Truck Based on Image Recognition. Journal of Civil Engineering and Management, 36, 170-177.

Liao, Z. P., Ding, H., Xia, Y., \& Ma, F. (2018). The Data-Based Study on the Information System of Risk Assessment in the City's Tunnel. Modern Tunnelling Technology, 55, 850-854.

Liu, J. B., Huang, Y. P., Wang, S. C., Zhao, X. X., \& Zhou, Q. (2019). Rail Fastener Defect Detection Method for Multi Railways Based on Machine Vision. China Railway Science, 40, 27-35.

Liu, Z. H., \& Zhang, Q. L. (2014). Research Overview of Big Data Technology. Journal of Zhejiang University (Engineering Science), 48, 957-972.

Ma, G. X., Han, Y., Sun, J. N., Sun, J. N., Gu, Y. Q., \& Sun, H. (2018). Design and Implementation of Automatic Safety Inspection System for External Scaffold Based on UAV. Journal of Civil Engineering and Management, 35, 169-174.

Ma, H. W., \& Nie, Z. H. (2015a). Recent Advances and Review of Bridge Safety Monitoring. Mechanics in Engineering, 37, 161-170 + 181 .

Ma, Z. L., Liu, S. L., \& Liu, Z. (2015b). Big Data Techniques and Its Applications in Civil Engineering. Journal of Information Technology in Civil Engineering and Architecture, $7,45-49$.

Pei, Y. L., \& Zhang, T. (2019). Vehicle Number Plate and Vehicle Identification System Based on Artificial Intelligence. Highway, 64, 277-281.

Phung, M. D., Quach, C. H., Dinh, T. H., \& Ha, Q. (2017). Enhanced Discrete Particle Swarm Optimization Path Planning for UAV Vision-Based Surface Inspection. Automation in Construction, 81, 25-33. https://doi.org/10.1016/j.autcon.2017.04.013

Rao, X. K., Ma, R., Zhang, L., \& Yi, C. Z. (2019). Big Data Safety Management Platform for Dyke Engineering Based on Artificial Intelligence: Research and Implementation. Journal of Yangtze River Scientific Research Institute, 36, 104-110.

Ren, X. Y., Huang, C., Tian, C. S., \& Wang, Q. (2018). Architecture of Mass Data Processing Platform for Building Cracks. Architecture Technology, 49, 61-64.

Tu, C. F., Liu, Z. J., Zhang, G., Zhou, L. C., Chen, Y. T., Cheng, N., Gu, J. W., Dong, S. B., Deng, Z. H., Wang, Y., \& Tang, L. Q. (2017). Processing Technique and Application of Big Data Oriented to Long-Term Bridge Health Monitoring. Journal of Experimental Mechanics, 32, 652-663. 
Wang, W., Li, M., \& Xu, H. (2018). Tunnel Safety Monitoring System Based on Video Big Data. China ITS Journal, S1, 162-164.

Wang, Y. W., \& Wu, Y. D. (2012a). Research on Smart Construction Concept and It's Supportive System. China Civil Engineering Journal, 45, 241-244.

Wang, Y. W., \& Wu, Y. D. (2012b). Research on the Theory and Key Technology of Intelligent Construction. Science \& Technology Progress and Policy, 29, 13-16.

Wu, Z. X., Han, P., Zheng, S. Q., \& Huang, H. Y. (2018). Design and Implementation of Bridge Health Monitoring and Maintenance Platform. Computer Applications and Software, 35, 109-114.

Xu, J. Q., Liu, X. Y., Su, Y. F., \& Huang, Q. Y. (2019). Study on Dynamic Risk Assessment Method of Building Fire Based on Bayesian Network. Journal of Safety Science and Technology, 15, 138-144.

Yang, Q., Wu, G. N., \& Wang, L. Z. (2017). Big Data: A New Perspective of the Engineering Project Management Driven by Data. Systems Engineering Theory \& Practice, 37, 710-719.

Yu, H., He, D.-N., Wang, G.-Y., Li, J., \& Xie, Y.-F. (2019). Big Data for Intelligent Decision Making. Acta Automatica Sinica, 20, 1-19.

Yu, Y. T., Guo, H. L., Ding, Q. H., Li, H., \& Skitmore, M. (2017). An Experimental Study of Real-Time Identification of Construction Workers' Unsafe Behaviors. Automation in Construction, 82, 193-206. https://doi.org/10.1016/j.autcon.2017.05.002

Zeng, H. (2014). Application of Big Data Mining in Project Management. Science \& Technology Progress and Policy, 31, 46-48.

Zeng, H. (2017). Research on Intelligent Helmets System for Engineering Construction. Harbin: Harbin Institute of Technology.

Zhang, Y. Y., Lin, J. R., \& Zhang, J. P. (2018). Present and Future of Integrated Applications of BIM, Cloud Computing, Big Data and Internet of Things. Journal of Graphics, 39, 806-816.

Zhi, P., Shi, T. Y., Wang, H. P., \& Wang, W. Q. (2018). BIM Techniques for Construction Management of the Qinghuayuan Tunnel on the Beijing-Zhangjiakou High-Speed Railway. Modern Tunnelling Technology, 55, 53-58.

Zhou, C., Ding, L. Y., Skibniewski, M. J., Luo, H. B., \& Zhang, H. T. (2018). Data Based Complex Network Modeling and Analysis of Shield Tunneling Performance in Metro Construction. Advanced Engineering Informatics, 38, 168-186. https://doi.org/10.1016/j.aei.2018.06.011

Zhu, Y. M. (2017). Data-Driven Fire Risk Prediction System. Fire Science and Technology, 36, 1011-1013.

Zhu, Z. H., Ren, X. N., \& Chen, Z. (2017). Integrated Detection and Tracking of Workforce and Equipment from Construction Jobsite Videos. Automation in Construction, 81, 161-171. https://doi.org/10.1016/j.autcon.2017.05.005 